\title{
Evaluation of Perienhancing Area in Differentiation between Glioblastoma and Solitary Brain Metastasis
}

\author{
Jureerat Thammaroj, Nattha Wongwichit, Arunnit Boonrod*
}

\begin{abstract}
Purpose: Accurate differential diagnosis between glioblastoma and brain metastasis is important. We aimed to differentiate these tumors by evaluation of the perienhancing area. Materials and Methods: Thirty patients with glioblastoma and solitary brain metastasis were included. The diameters of perienhancing and enhancing areas were measured, and the percentage of enhancing area was calculated. We measured Apparent diffusion coefficient (ADC) of perienhancing and enhancing areas. Intratumoral necrotic areas were measured. Results: The enhancing area of glioblastoma was $56.61 \%$ and metastasis was $42.55 \%(\mathrm{p}=0.08)$. The ADC values of the perienhancing part of GBM was 0.7 and metastasis was $0.79(\mathrm{p}=0.052)$. The ADC value of the enhancing part of the GBM was 0.82 and metastasis was 0.8 (p-value $=0.72)$. The intratumoral necrotic area of glioblastoma $\left(152.25 \mathrm{~mm}^{3}\right)$ was higher than in metastasis $\left(0 \mathrm{~mm}^{3}\right)(\mathrm{p}$-value $=0.003)$ with a cutoff area of $11.8 \mathrm{~mm}^{2}$. Conclusion: The ADC values of the perienhancing area were lower in glioblastoma with a near-significant $\mathrm{p}$-value. Other perienhancing parameters demonstrated no significant difference between both tumors. The intratumoral necrotic area of glioblastoma is larger than metastasis.
\end{abstract}

Keywords: Perienhancing area- differentiation- glioblastoma- GBM- solitary brain metastasis- intratumoral necrosis

Asian Pac J Cancer Prev, 21 (9), 2525-2530

\section{Introduction}

Glioblastoma and brain metastasis are two of the most common brain tumors in adults. Accurate imaging diagnosis is important because of the differing treatments for these conditions (Stark et al., 2012; Wu et al., 2015). Glioblastoma and solitary brain metastasis can sometimes appear similar on conventional MRI, which can make the definite diagnosis difficult. Both glioblastoma and brain metastasis demonstrate heterogeneous signal in conventional MRI with various types of enhancement surrounded by a perienhancing edematous area (Sentürk, Oğuz, and Cila, 2009; Hakyemez et al., 2010; Halshtok Neiman et al., 2013; Wu et al., 2015).

Glioblastoma and metastasis differ in degrees of perilesional edema. Few previous studies have shown significant differences between the ratio of peritumoral edema and enhancing tumor (Maurer et al., 2013; Baris et al., 2016). Perilesional edema was greater in brain metastasis than in glioblastoma.

Diffusion-weighted image (DWI) and apparent diffusion coefficient (ADC) values are known to help differentiate between tumor types because they represent the movement of water molecules in vivo (Hagmann et al., 2006; Phuttharak et al., 2018). Several studies have yielded inconclusive results when trying to make a differential diagnosis between glioblastoma and brain metastasis, especially when relying on values measured at the enhanced part of the tumor (Calli et al., 2006; Guzman et al., 2008; Lee et al., 2011; Tsougos et al., 2012; Maurer et al., 2013; Lemercier et al., 2014; Dawoud, Sherif, and Eltomey, 2014). Even though preliminary results are inconclusive, we believe DWI is a potential tool for differential diagnosis between these two tumors. In pathology, glioblastoma has combined peritumoral infiltration and edema but brain metastasis has pure perilesional vasogenic edema (Pekmezci and Perry, 2013; Louis et al., 2016). Accordingly, ADC values of the perilesional area should be different between these two groups: glioblastoma should have lower ADC values than metastasis.

In our study, we evaluated two main aspects of the perihancing area. We measured the maximal diameter of the area and also the ADC values relative to the enhancing area, to differentiate between glioblastoma and solitary metastasis.

\section{Materials and Methods}

\section{Patients}

Thirty consecutive patients with histopathologically proven solitary brain metastasis $(\mathrm{n}=15)$ or glioblastoma 
$(n=15)$ who underwent pre-treatment MRI of the brain using DWI, between 1 January 2011 and 1 December 2016, were retrospectively reviewed. One patient had two lesions pathologically proven to be glioblastoma: the total number of tumors included was therefore 31 . The study was approved by the local Institutional Review Board of ethical issues with a waiver of informed consent.

\section{Imaging techniques}

Seven patients were imaged using a 1.5T MR scanner (MAGNETOM Aera; Siemens, Erlangen, Germany) and twenty-one patients were imaged with a 3T MR scanner (Phillips Achieva; Philips, Best, the Netherlands). Routine MR pulse sequences for the 1.5T scanner include: sagittal and axial T1-weighted image spin echo [T1WI SE] [TR 450-600, TE 8-10], axial T2-weighted image turbospin echo [T2WI turboSE] [TR 3500-5000, TE 80-100], axial fluid-attenuated inversion recovery [FLAIR] [TR 9000, TE 120, TI 2300], and coronal T2GRE [TR 700-800, TE 15-35, FA 20]. Routine MR pulse sequences for the $3 \mathrm{~T}$ scanner include: sagittal T1WI 3D TFE [TR shortest, TE shortest, FA 8], axial T2WI TSE [TR 3000-5000, TE 80], axial FLAIR [TR 11000, TE 125, TI 2800], and coronal T2GRE [TR 700-800, TE 15-35, FA 18]. Imaging parameters of DWI were as follows: 1819-8000/85-93 [TR/TE] with diffusion sensitivities $b=0$ and $b=1,000$ $\mathrm{s} / \mathrm{mm}^{3}$ for both scanners. The diffusion gradients were applied sequentially in three orthogonal directions to generate 2 sets of axial DW images. The ADC maps were automatically generated from the datasets of DW images using the operating console (Synapse 3D workstation, Fujifilm Medical Systems, USA) and ADC values were calculated. After administration of gadolinium-based contrast agent, multiplanar T1WI was performed. In all sequences, the field of view was $22-24 \mathrm{~cm}$. and the section thickness was $5 \mathrm{~mm}$.

\section{Image analysis}

All MRIs were analyzed by a senior neuroradiologist with 20 years' experience in neuroimaging. We defined perienhancing areas as the non-enhancing hypersignal T2WI areas surrounding the enhancing tumor. At the perienhancing area, the maximal diameter in axial T2WI was measured, and the minimum ADC areas were visually inspected and five regions of interest (ROIs) were drawn in different places. At the enhancing area, the maximal diameter was measured in axial view on post-contrast T1WI and five ROIs for ADC values were measured (Figure 1 and 2). The percentage of the enhancing area to the perienhancing area was calculated. The ADC ratio was calculated by dividing the mean of five perienhancing $\mathrm{ADC}$ values by the mean of five enhancing ADC values.

We also recorded the extent of intratumoral necrotic areas $\left(\mathrm{mm}^{2}\right)$. We defined these areas as the non-enhancing hypersignal T2WI area within the enhancing tumor if they showed increased diffusion on DWI and ADC maps (hypersignal on both DWI and ADC maps). The area was calculated from free-hand ROI drawings on the ADC map (Figure 3) If more than one necrotic area was identified, each area was measured at its largest extent and values were summed.

\section{Statistical analysis}

Statistical analysis was performed using Stata statistical software package, version 10. Demographic data of all patients and the site of the primary tumor of the metastasis patients were interpreted by descriptive analysis. To determine the difference between metastasis and glioblastoma, we analyzed the ratios of diameters, the ADC values, and ADC ratios using two-sample Wilcoxon rank-sum tests (Mann-Whitney). A receiver operating characteristic (ROC) analysis was used to determine the cutoff value, sensitivity and specificity of the intratumoral necrotic area as a tool for differentiation between glioblastoma and brain metastasis. A p-value of $<0.05$ indicated a statistically significant difference.

\section{Results}

Of the thirty patients included, sixteen were males $(53.3 \%)$ and fourteen were females $(46.7 \%)$. Their ages ranged from 7 to 71 years $(48 \pm 18.5$ years, mean $\pm \mathrm{SD})$. Both glioblastoma and metastasis groups had fifteen patients, eight males and seven females. The mean age of metastasis patients was $58( \pm 10)$ years and the mean age of glioblastoma patients was $38( \pm 20)$ years. The primary tumors producing solitary metastasis were lung cancer ( 7 patients, $46.7 \%$ ), head and neck cancer ( 3 patients, $20 \%$ ), breast cancer ( 2 patients, $13.3 \%$ ), neuroendocrine tumor (1 patient, 6.7\%), renal angiomyelolipoma (1 patient, 6.7\%), and cholangiocarcinoma (1 patient, $6.7 \%)$.

Table 1 provides summary statistics (medians and percentiles) on the extent of enhancing areas, ADC values of the perienhancing and enhancing areas (Figure 1 and 2), $\mathrm{ADC}$ ratios (Figure 2) and intratumoral necrotic areas

Table 1. Comparisons between Glioblastoma and Metastasis in Terms of ADC Values, ADC Ratios, Intratumoral Necrotic Areas and Percentage of Enhancing Areas

\begin{tabular}{|c|c|c|c|}
\hline & Median & $\mathrm{P} 25, \mathrm{P} 75$ & p-value \\
\hline \multicolumn{4}{|c|}{ Perienhancing ADC values $\left(\times 10^{-3} \mathrm{~mm}^{2} / \mathrm{s}\right)$} \\
\hline GBM & 0.7 & $0.59,0.76$ & $0.052 *$ \\
\hline Metastasis & 0.79 & $0.74,0.92$ & \\
\hline \multicolumn{4}{|c|}{ Enhancing ADC values $\left(\times 10^{-3} \mathrm{~mm}^{2} / \mathrm{s}\right)$} \\
\hline GBM & 0.82 & $0.73,0.94$ & 0.72 \\
\hline Metastasis & 0.8 & $0.72,1.11$ & \\
\hline \multicolumn{4}{|l|}{$\mathrm{ADC}$ ratio $^{\mathrm{a}}$} \\
\hline GBM & 0.87 & $0.67,1.05$ & 0.52 \\
\hline Metastasis & 0.95 & $0.73,1.03$ & \\
\hline \multicolumn{4}{|c|}{ Area of tumoral necrosis $\left(\mathrm{mm}^{2}\right)$} \\
\hline GBM & 152.25 & $28.2,362.9$ & $0.003 * *$ \\
\hline Metastasis & 0 & $0,103.6$ & \\
\hline \multicolumn{4}{|c|}{ Percentage of enhancing area } \\
\hline GBM & 56.6 & $48.5,74.3$ & 0.08 \\
\hline Metastasis & 42.6 & $29.6,66.7$ & \\
\hline
\end{tabular}

${ }^{a} \mathrm{ADC}$ ratio was calculated by dividing the perienhancing $\mathrm{ADC}$ value by the enhancing area ADC value; *, Near significant p-value; **, Significant p-value 

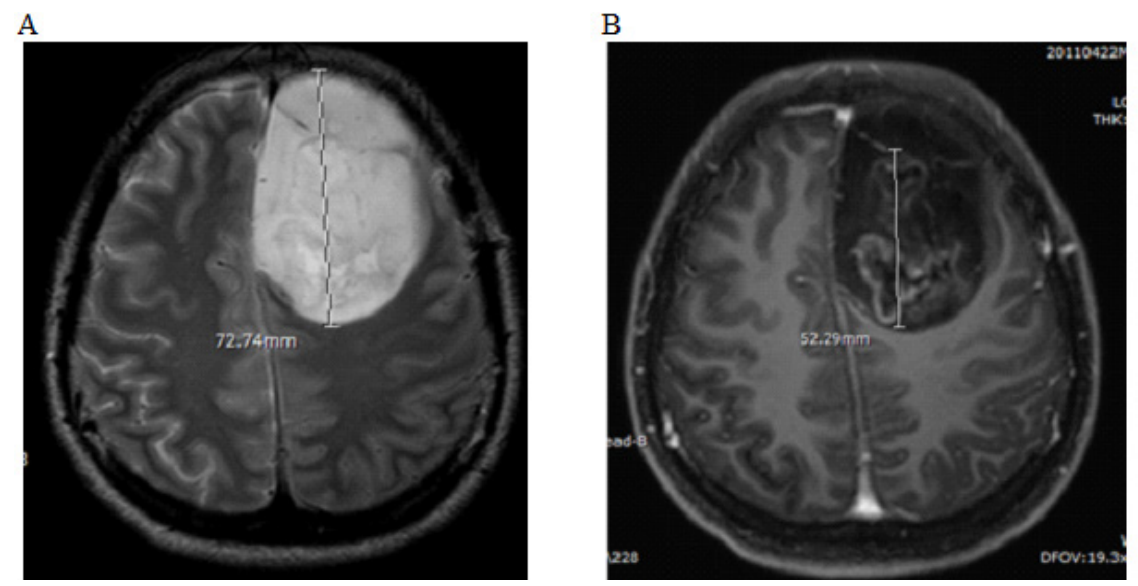

Figure 1. A 27-Year-Old Woman with a Glioblastoma at Left Frontal Lobe. T2WI (A) and post contrast T1WI (B) showed a heterogeneous hypersignal T2WI mass with irregular ring enhancement. The maximal diameters of perienhancing and enhancing areas were 72.7 and $52.3 \mathrm{~mm}$, respectively. The percentage of enhancing area was $71.9 \%$

(Figure 3) of the glioblastoma and metastatic groups. The median of ADC values $\left(\times 10^{-3} \mathrm{~mm}^{2} / \mathrm{s}\right.$, ) of perienhancing areas of glioblastomas and metastasis were 0.7 and 0.79 ( $\mathrm{p}$-value $=0.052)$. The median of ADC values $\left(\mathrm{x} 10^{-3} \mathrm{~mm}^{2} / \mathrm{s}\right.$, ) of enhancing areas of glioblastomas and metastasis were 0.82 and 0.8 ( $\mathrm{p}$-value $=0.72$ ). The median of ADC ratios, calculated by dividing the perienhancing $\mathrm{ADC}$ value by the enhancing area ADC value, of glioblastomas and metastasis were 0.87 and 0.95 $(p$-value $=0.52)$. The median of intratumoral necrotic areas $\left(\mathrm{mm}^{2}\right)$ of glioblastomas and metastasis were 152.25 and 0 (p-value $=0.003)$.

A receiver operating characteristic (ROC) curve was used to analysis the intratumoral necrotic area for differentiation of glioblastoma and metastasis. The area under the ROC curve was 0.80 (95\% CI 0.64, 0.95). When the cutoff value for the necrotic area was set as $11.8 \mathrm{~mm}^{2}$, sensitivity $(87.5 \%)$ and specificity $(66.67 \%)$ were obtained (Figure 4).
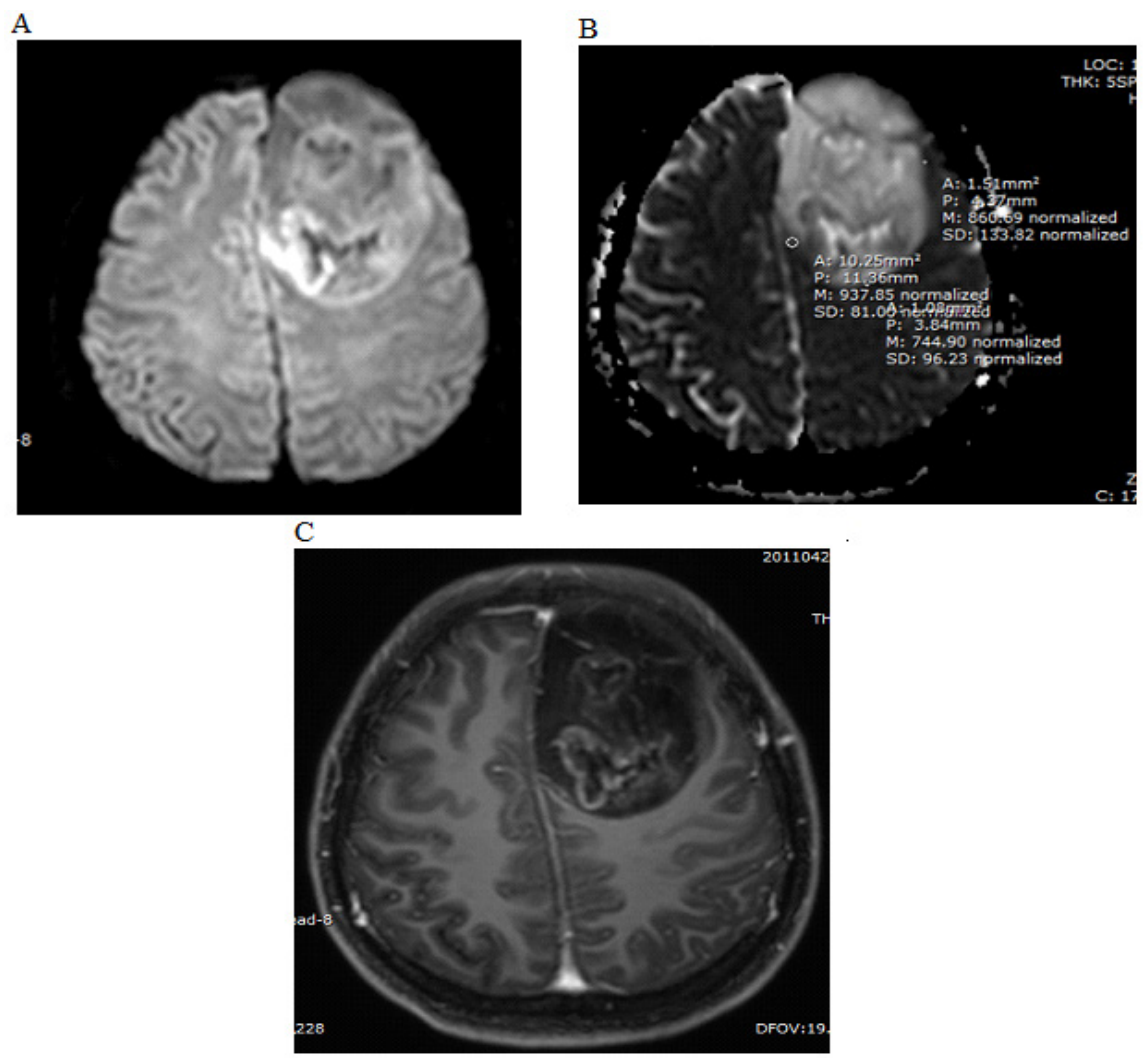

Figure 2. The Same Patient. DWI (A), ADC Map (B) and Axial Post Contrast T1WI (C) Demonstrated Restricted Diffusion Areas at Perienhancing Area. At this image, three ROIs were drawn on the ADC map (B) at the area with minimal ADC value. The measured ADC values in this image were $0.937,0.744$ and $0.86 \times 10^{-3} \mathrm{~mm}^{2} / \mathrm{s}$. The mean ADC value of the perienhancing area of this case was $0.86 \times 10^{-3} \mathrm{~mm}^{2} / \mathrm{s}$. Post-contrast image $(\mathrm{C})$ showed that no enhancing part was included in the measurement of perienhancing area. 

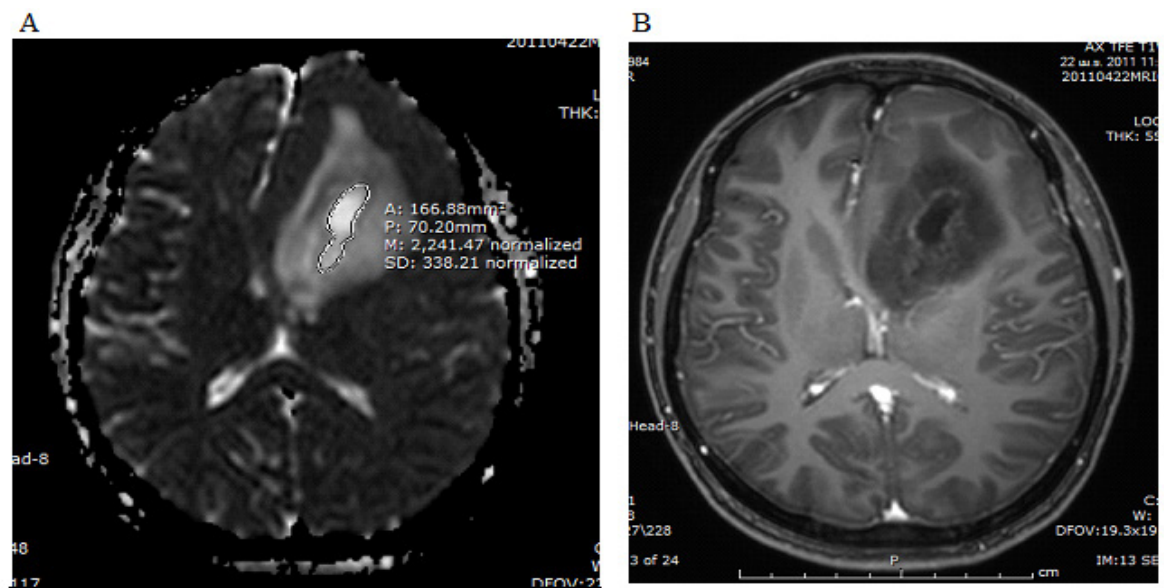

Figure 3. The Same Patient, ADC Map (A) and Post Contrast T1WI (B) Demonstrated an Intratumoral Non-Enhancing Area with Increased Diffusion, Representing the Intratumoral Necrotic Area. ROIs were drawn free-hand on the ADC map. The intratumoral necrotic area in this image was $166.9 \mathrm{~mm}^{2}$ and the sum of intratumoral necrotic area measurements in this patient was $561.4 \mathrm{~mm}^{2}$.

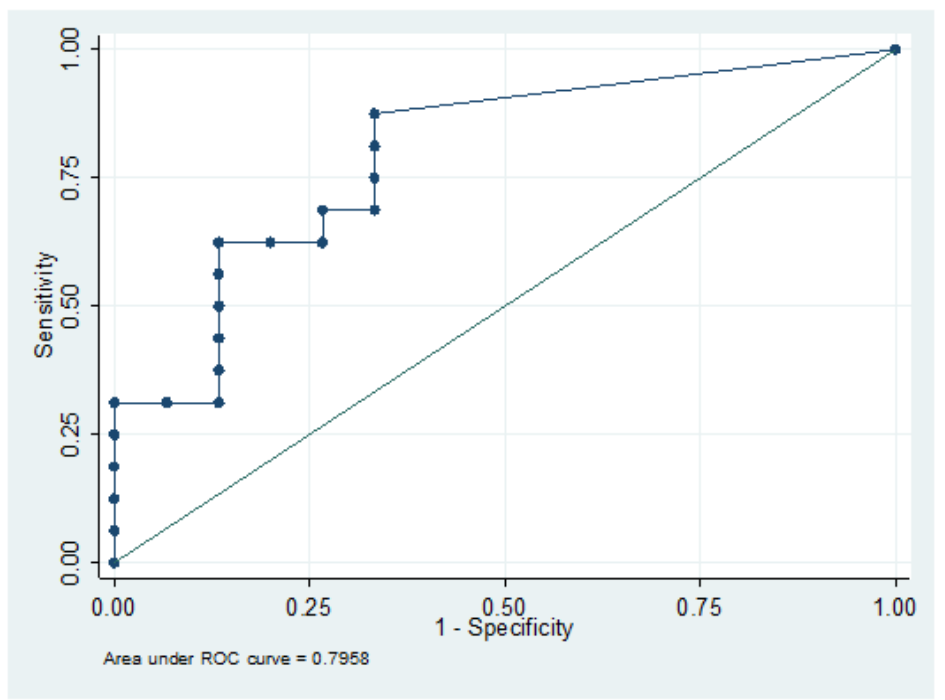

Figure 4. Receiver Operating Characteristic (ROC) Curve of Intratumoral Necrotic Area for Differentiation of Glioblastoma from Metastasis. The area under the ROC curve was 0.8 (95\% CI 0.64, 0.95). When the cutoff necrotic area was set as $11.8 \mathrm{~mm}^{2}$, sensitivity $(87.5 \%)$ and specificity $(66.67 \%)$ were obtained.

\section{Discussion}

Differentiation between glioblastoma and brain metastasis is significant for the planning of both diagnostic workup and treatment. Accurate pre-treatment diagnosis will be a benefit to both the patients and the clinicians.

Heterogeneous cell structures were found in the solid tumoral parts of both glioblastoma and metastasis tissues in histology. However, at the peritumoral area, a glioblastoma typically has peritumoral infiltration, but a brain metastasis does not (Pekmezci and Perry, 2013). Several studies have shown no significant difference in peritumoral ADC values between glioblastoma and metastases (Calli et al., 2006; Lee et al., 2011; Maurer et al., 2013; Dawoud et al., 2014). Few studies have shown lower peritumoral ADC values in glioblastoma as compared to metastasis (Guzman et al., 2008; Lemercier et al., 2014; Blystad et al., 2017). We found median ADC values in the non-enhancing area at the edge of the tumor to be lower in metastases than in glioblastoma, with a near-significant $p$-value $(p$-value $=0.052)$. This finding may help to distinguish between GBM and metastases and supports the hypothesis that a glioblastoma involves peritumoral infiltration but a brain metastasis does not (Chilla et al., 2015; Celik, 2016).

An enhancing mass with extensive perilesional brain edema is a classic characteristic of brain metastasis. However, the perilesional edema in metastasis can vary from minimal to extensive (Sharma et al., 2013). A previous study found a statistically significant difference in the ratio of enhancing tumor to perilesional edema (Maurer et al., 2013; Baris et al., 2016), whereas we found no such difference.

Intratumoral necrosis is believed to occur because of insufficient blood supply within a rapidly growing malignant tumor. However, for glioblastoma, microvascular hyperplasia is found around the necrotic area, which may represent an prothrombotic or vaso-occlusive state within the tumor (Altmanet al., 2007) This may cause more intratumoral necrosis in glioblastoma as compared to brain 
metastasis, as reflected in the significant difference in our study. The intratumoral necrotic area in glioblastoma was higher than in metastases ( $p$-value $=0.003$ ) with the cutoff point of $11.8 \mathrm{~mm}^{3}$ (sensitivity of $87.5 \%$ and specificity of $66.67 \%$ ).

This study had a few limitations. First, the study was retrospective in nature. Second, two different MR scanners (1.5T and 3T) were used, with slightly different technique, which may reduce comparability of the ADC values. Third, the sample size was small with a considerable age difference between the glioblastoma and metastasis group. Further prospective study with larger populations and uniform MR technique are required to extend the results.

In conclusion, the $\mathrm{ADC}$ values of the perienhancing area were lower in glioblastoma than in solitary brain metastases with a near-significant p-value. Other perienhancing parameters, including $\mathrm{ADC}$ ratio and percentage of enhancement, demonstrated no significant differences between both tumors. The intratumoral necrotic area of glioblastoma is larger than metastasis with the cutoff value of $11.8 \mathrm{~mm}^{2}$.

\section{Acknowledgments}

We would like to acknowledge Clinical Epidemiology Unit, Khon Kaen University, Thailand.

\section{Ethical approval}

All procedures performed in reports involving human participants were in accordance with the ethical standards of the institutional and/or national research committee and with the 1964 Helsinki declaration and its later amendments or comparable ethical standards. For this type of report formal consent is not required.

\section{Informed consent}

Informed consent was not obtained. Office of The Khon Kaen University Ethics Committee in human research KKU EC approved this study.

\section{Contributors statement}

J.T. contributions to the conception or design of the work, analysis and final approval. N.W. contributions to the conception or design of the work, and analysis. A.B. ensures that the study receives all necessary IRB approvals, contributions to the conception or design of the work, revising it critically for important intellectual content and final approval.

\section{Conflict of Interest}

The authors declare that they have no conflict of interest.

\section{References}

Altman DA, AtkinsonDS, Brat DJ (2007). Glioblastoma Multiforme. RadioGraphics, 27, 883-8.

Baris MM, Celik AO, Gezer NS, Ada E (2016). Role of mass effect, tumor volume and peritumoral edema volume in the differential diagnosis of primary brain tumor and metastasis. Clin Neurol Neurosurg, 148, 67-71.
Blystad I, Warntjes JBM, Smedby Ö, et al (2017). Quantitative MRI for analysis of peritumoral edema in malignant gliomas. PLoS One, 12, e0177135.

Calli C, Kitis O, Yunten N, et al (2006). Perfusion and diffusion MR imaging in enhancing malignant cerebral tumors. Eur J Radiol, 58, 394-403.

Celik A (2016). Effect of imaging parameters on the accuracy of apparent diffusion coefficient and optimization strategies. Diagn Interv Radiol, 22, 101-7.

Chilla GS, Tan CH, Xu C, Poh CL (2015). Diffusion weighted magnetic resonance imaging and its recent trend-a survey. Quant Imaging Med Surg, 5, 407-22.

Dawoud MAE, Sherif MF, Eltomey MA (2014). Apparent diffusion coefficient and Magnetic resonance spectroscopy in grading of malignant brain neoplasms. Egypt J Radiol Nuclear Med, 45, 1215-22.

Guzman R, Altrichter S, El-Koussy M, et al (2008). Contribution of the apparent diffusion coefficient in perilesional edema for the assessment of brain tumors. J Neuroradiol, 35, 224-9.

Hagmann P, Jonasson L, Maeder P, et al (2006). Understanding diffusion MR imaging techniques: from scalar diffusion-weighted imaging to diffusion tensor imaging and beyond. Radiographics, 26, 205-23.

Hakyemez B, Erdogan C, Gokalp G, Dusak A, Parlak M.(2010) Solitary metastases and high-grade gliomas: radiological differentiation by morphometric analysis and perfusion-weighted MRI. Clin Radiol, 65, 15-20.

Halshtok Neiman O, Sadetzki S, Chetrit A, et al (2013). Perfusion-weighted imaging of peritumoral edema can aid in the differential diagnosis of glioblastoma mulltiforme versus brain metastasis. Isr Med Assoc J, 15, 103-5.

Lee EJ, terBrugge K, Mikulis D, et al (2011). Diagnostic value of peritumoral minimum apparent diffusion coefficient for differentiation of glioblastoma multiforme from solitary metastatic lesions. AJR Am J Roentgenol, 196, 71-6.

Lemercier P, Paz Maya S, Patrie JT, Flors L, Leiva-Salinas C (2014). Gradient of apparent diffusion coefficient values in peritumoral edema helps in differentiation of glioblastoma from solitary metastatic lesions. AJR Am J Roentgenol, 203, 163-9.

Louis DN, Perry A, Reifenberger G, et al (2016). The 2016 World Health Organization Classification of Tumors of the Central Nervous System: a summary. Acta Neuropathol, 131, 803-20.

Maurer MH, Synowitz M, Badakshi H, et al (2013). Glioblastoma multiforme versus solitary supratentorial brain metastasis: differentiation based on morphology and magnetic resonance signal characteristics. Rofo, 185, 235-40.

Pekmezci M, Perry A (2013). Neuropathology of brain metastases. Surg Neurol Int, 4, 245-55.

Phuttharak W, Boonrod A, Thammaroj J, Kitkhuandee A, Waraasawapati S (2018). Preoperative MRI evaluation of meningioma consistency: A focus on detailed architectures. Clin Neurol Neurosurg, 169, 178-84.

Sentürk S, Oğuz KK, Cila A (2009). Dynamic contrastenhanced susceptibility-weighted perfusion imaging of intracranial tumors: a study using a 3T MR scanner. Diagn Interv Radiol, 15, 3-12.

Sharma V, Prabhash K, Noronha V, Tandon N, Joshi A (2013). A systematic approach to diagnosis of cystic brain lesions. South Asian J Cancer, 2, 98-101.

Stark AM, van de Bergh J, Hedderich J, Mehdorn HM, Nabavi A (2012). Glioblastoma: Clinical characteristics, prognostic factors and survival in 492 patients. Clin Neurol Neurosurg, 114, 840-5.

Tsougos I, Svolos P, Kousi E, et al (2012). Differentiation of glioblastoma multiforme from metastatic brain tumor 
Jureerat Thammaroj et al

using proton magnetic resonance spectroscopy, diffusion and perfusion metrics at $3 \mathrm{~T}$. Cancer Imaging, 12, 423-36.

Wu C-X, Lin G-S, Lin Z-X, et al (2015). Peritumoral edema shown by MRI predicts poor clinical outcome in glioblastoma. World J Surg Oncol, 13, 97.

\section{c) (1) (8)}

This work is licensed under a Creative Commons AttributionNon Commercial 4.0 International License. 